\title{
THE SO-CALLED ANTIFLARE STARS
}

\author{
A. F. PUGACH \\ Main Astronomical Observatory, Ukrainian Academy of Sciences, Kiev, U.S.S.R.
}

\begin{abstract}
There seems to exist a small subgroup of irregular variables with light curves similar to $\mathrm{R} \mathrm{CrB}$ stars but with a shorter time scale. These stars have smallamplitude light variations around a 'normal brightness', but this is interrupted by Algol-like minima of about 0.8 to $2^{m} .0$ at irregular intervals. The duration of the minimum phase is several percent of the total time. While at minimum the variables also show rapid light variation of greater amplitude. Moreover, RZ Psc shows a flare activity, which strongly resembles that of UV Cet stars. The minimum phase is followed by a rapid recovery to the 'normal brightness'.
\end{abstract}

To be an antiflare object a star must satisfy certain conditions.

(1) The probability density function (or frequency distribution) of magnitudes should have a great positive asymmetry. As a consequence of this ' $T$ ' is much greater than ' $t$ ', so are ' $A$ ' and ' $a$ ' (see Figure 1).

(2) Distribution of magnitudes at maximum is Gaussian.

(3) Brightness change rate during an antiflare is about $0 . m$ an hour. Several examples are given in Figures 1-3. A list of 'antiflare' variables and some stars suspected to belong to this class are given in Table $\mathrm{I}$.

TABLE I

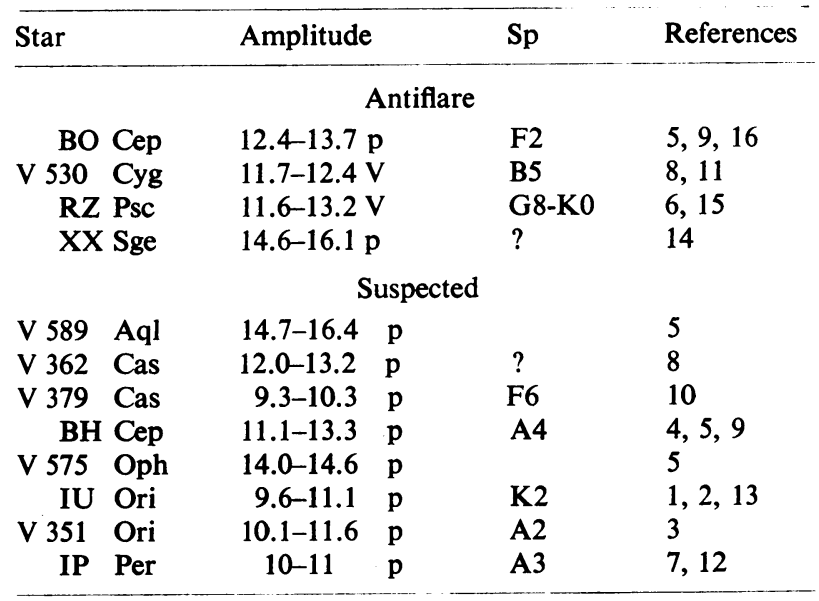

\section{References to Table I}

[1] Bateson, M. F.: 1972, New Zealand Circ., No. 191.

[2] Eggen, O. J., 1973, Publ. Astron. Soc. Pacific 85, 42.

[3] Filin, A. Aj.: 1951, Astron. Circ., No. 111, 10.

[4] Hoffmeister, C.: 1936, Astron. Nachr. 259, 37.

[5] Hoffmeister, C.: 1949, Astron. Nachr. 278, 24.

[6] Karetnikov, V. G. and Pugach, A. F.: 1973, Info. Bull. Var. Stars, No. 783.

[7] Kippenhahn, R.: 1953, Astron. Nachr. 281, 155.

[8] Meinunger, L.: 1966, Mitt. Veränd. Sterne 3, 161. 
[9] Mosidze, L. N.: 1969, Abastumani Bull., No. 37, 18.

[10] Nikulina, T. G.: 1959, Astron. Circ., No. 207, 16.

[11] Pugach, A. F.: 1970, manuscript deposited in VINITI (Moscow), register 1982-70.

[12] Solovyev, A. V.: 1954, Astron. Circ., No. 149, 20.

[13] Solovyev, A. V. and Erleskova, G. E.: 1962, Tadjik Bull., No. $34,3$.

[14] Tsesevich, V. P. and Dragomiretskaya, B. A.: 1973, RW Aurigae Stars.

[15] Wenzel, W.: 1956, Mitt. Veränd. Sterne, No. 217.

[16] Wenzel, W.: 1961, Veröff. Sonneberg 5, 60.

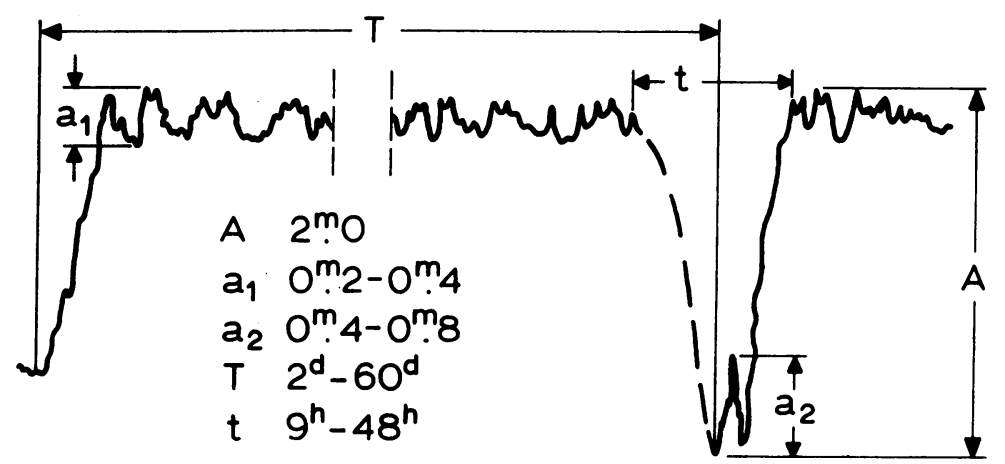

Fig. 1. Schematic light curve of an anti-flare star.

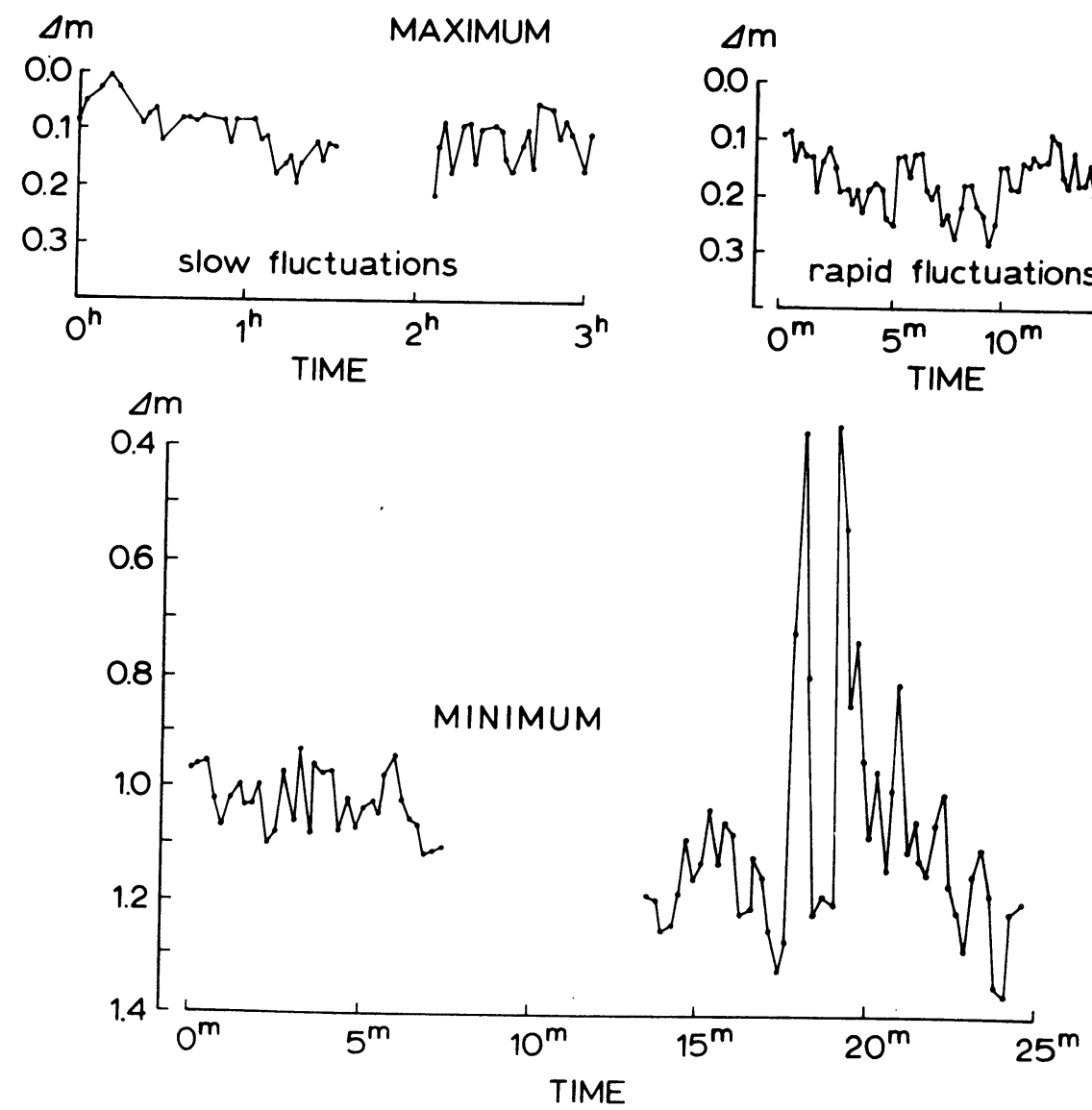

Fig. 2. Fragments of the light curve of RZ Psc. 


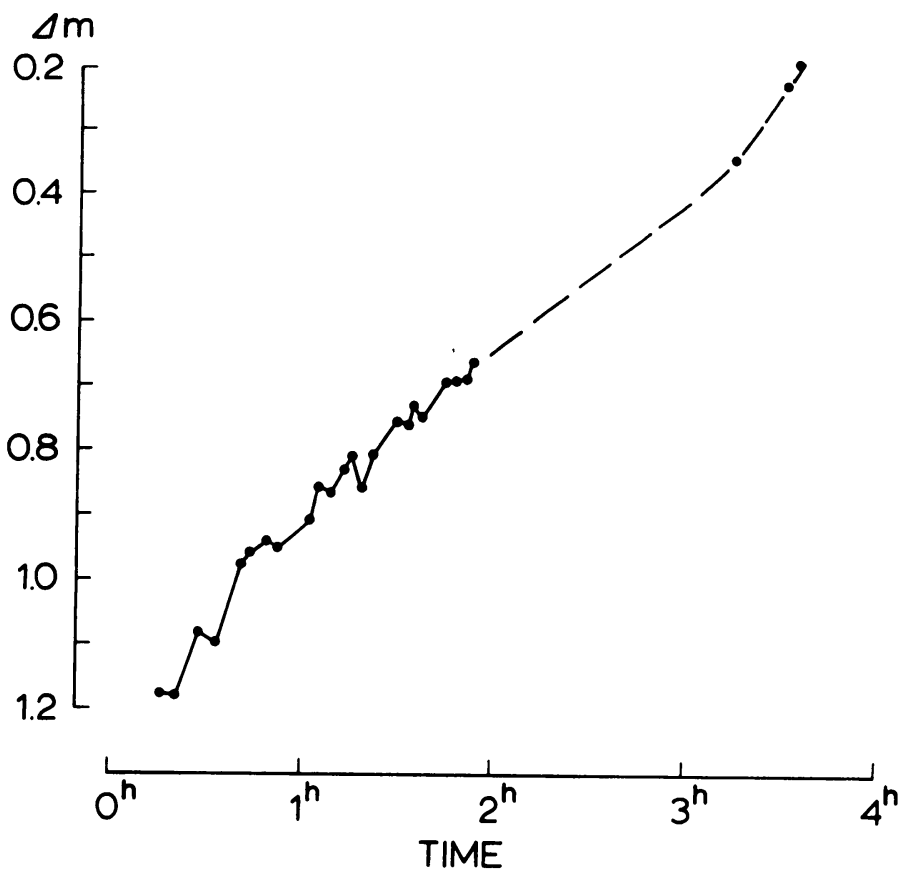

Fig. 3. Rising branch of RZ Psc.

\section{DISCUSSION}

W. Wenzel: What is the wavelength dependence of the light variation of PZ Psc? A. F. Pugach: It seems that PZ Psc is getting bluer during minimum. 